\title{
Estrategias de Afrontamiento y Nivel de Resiliencia Presentes en Adultos Jóvenes Víctimas del Conflicto Armado Colombiano: Un Estudio Correlacional
}

\section{Coping Strategies and Resilience Level of Young Adult Victims of the Colombian Armed Conflict: A Correlational Study}

\author{
Ara Mercedes Cerquera Córdoba, Yeferson Jahir Matajira Camacho y Arbey J Peña Peña \\ Universidad Pontificia Bolivariana
}

\begin{abstract}
El conflicto armado en Colombia ha generado por décadas afectaciones a la población. El objetivo del presente estudio fue analizar la relación entre las estrategias de afrontamiento y entre estas y el nivel de resiliencia de una muestra de adultos jóvenes víctimas del conflicto armado colombiano. Con un enfoque cuantitativo de alcance descriptivo correlacional y muestreo por conveniencia en los municipios de Bucamaranga, Piedecuesta, Girón y Floridablanca de Colombia, se trabajó con 70 adultos jóvenes (23 hombres y 47 mujeres), utilizando una entrevista semiestructurada, la Escala de Estrategias de Coping-Modificada y la Escala de Resiliencia. Respecto a los resultados descriptivos, se identificó altas puntuaciones en las estrategias de afrontamiento de apoyo social, religión, reacción agresiva y expresión de la dificultad de afrontamiento y bajas puntuaciones en las estrategias de solución de problemas, espera, evitación emocional, búsqueda de apoyo profesional, evitación cognitiva, reevaluación positiva, negación y autonomía. En cuanto a la escala de resiliencia, se evidencia bajas puntuaciones en la muestra. Por último, se encontraron correlaciones entre las estrategias de afrontamiento y entre la evitación emocional con la ecuanimidad y sentirse bien solos, categorías de la resiliencia. Se discute que en esta situación de conflicto los sujetos presentan estrategias de afrontamiento no adaptativas que pueden influir en el desarrollo de trastornos psicopatológicos, al no potenciar la resiliencia.
\end{abstract}

Palabras clave: adultos jóvenes, conflicto armado, estrategias de afrontamiento, resiliencia

\begin{abstract}
The armed conflict in Colombia has affected the population for decades. The objective of the present study was to analyze the relationship among coping strategies and between these and the resilience level of a sample of young adult victims of the Colombian armed conflict. Adopting a quantitative approach of a descriptive and correlational nature and conducting a convenience sampling procedure in the Colombian municipalities of Bucamaranga, Piedecuesta, Girón, and Floridablanca, we worked with 70 young adults (23 men and 47 women), administering a semi-structured interview, the Coping Strategies Scale - Modified, and the Resilience Scale. Regarding the descriptive results obtained for the participants' coping strategies, high scores were identified in social support, religion, aggressive reaction, and expression of coping difficulties, while low scores were found in problem-solving, waiting, emotional avoidance, seeking professional support, cognitive avoidance, positive reevaluation, denial, and autonomy. Regarding the resilience scale, low scores were observed in the sample. Lastly, correlations were found among coping strategies and between emotional avoidance and equanimity and feeling good alone, two categories of resilience. It is argued that, in this conflict situation, subjects display non-adaptive coping strategies that can influence the development of psychopathological disorders by failing to enhance resilience.
\end{abstract}

Keywords: young adults, armed conflict, coping strategies, resilience

Por más de 50 años Colombia ha vivido en un ambiente continuo de violencia, donde todos los grupos involucrados en el conflicto armado han utilizado el terror como estrategia de guerra, desembocando en atentados contra los derechos humanos (Moreno Camacho \& Díaz Rico, 2016). Según estadísticas del Centro Nacional de Memoria Histórica (2013) y la Red Nacional de Información [RNI] (2019), a la fecha se han reportado 11.478 casos de abandono o despojo forzado de tierras, 47.743 víctimas directas por desapariciones forzadas, 7.513.774 víctimas de desplazamiento, 269.845 víctimas directas por homicidios y 32.946 víctimas directas de secuestro y tortura. Actualmente, la cifra total de personas registradas como víctimas es de 8.847.047 (RNI, 2019).

Ara Mercedes Cerquera Córdoba, Yeferson Jahir Matajira Camacho y Arbey Jair Peña Peña, Departamento de Psicología, Universidad Pontificia Bolivariana Seccional Bucaramanga, Colombia.

La correspondencia relativa a este artículo debe ser dirigida a Yeferson Jahir Matajira Camacho, Departamento de Psicología, Universidad Pontificia Bolivariana - Seccional Bucaramanga, Autopista Piedecuesta Kilómetro 7, Floridablanca, Santander, Colombia. E-mail: yeferson.matajira@upb.edu.co 
CERQUERA CÓRDOBA, MATAJIRA CAMACHO Y PEÑA PEÑA

Según considera Bello (2013) y otros autores, todas estas situaciones de violencia generan consecuencias físicas y psicológicas en las víctimas (Lira, 2010; McDonald, 2010; Rodríguez Díaz, 2006), entre estas, las afecciones más frecuentes son la depresión (Schweitzer, Greenslade \& Kagee, 2007), el estrés postraumático (Bell, Méndez, Martínez, Palma \& Bosch, 2012), la ansiedad, los ataques de pánico, los trastornos bipolares y el consumo de sustancias psicoactivas (Alejo, Rueda, Ortega \& Orozco, 2007). Lo anterior lo confirman estudios, prevaleciendo el trastorno de estrés postraumático y los trastornos depresivos y de ansiedad (Husain et al., 2011; Steel et al., 2009), incluso, algunos resaltan la coincidencia en la comorbilidad con estos trastornos (Bell et al., 2012).

En Colombia, Londoño, Romero y Casas (2012) encontraron una prevalencia de síntomas asociados a trastornos de ansiedad, trastorno de somatización, abuso de alcohol y trastornos de alimentación, mientras que Hewitt Ramírez et al. (2016) encontraron que las principales afecciones psicológicas son la paranoia, el consumo de alcohol, las alteraciones del estado anímico y los síntomas de estrés postraumático. Por otro lado, Constanza Zuluaga (2016) encontró síntomas como inhibición de la expresión de los sentimientos, desconfianza, indefensión, evitación, miedo, alteración en la construcción del proyecto de vida y alteraciones en la estructura y dinámica familiar (Corporación Acompañamiento Psicosocial y Atención en Salud Mental a Víctimas de Violencia Política, 2008).

Ahora bien, existen diferentes formas de enfrentar situaciones, las que se han denominado estrategias de afrontamiento (Huijts, Kleijn, van Emmerik, Noordhof \& Smith, 2012), las cuales permiten hacer frente a las distintas situaciones de violencia a las que la población está expuesta. Así, pues, las estrategias de afrontamiento son "aquellos esfuerzos cognitivos y conductuales constantemente cambiantes que se desarrollan para manejar las demandas específicas que son evaluadas como excedentes o desbordantes de los recursos del individuo" (Lazarus \& Folkman, 1986, p. 164).

Para Lazarus y Folkman (1986), las estrategias de afrontamiento se dividen en dos grupos. El primero, centrado en el problema, en el que el sujeto realiza una serie de acciones para cambiar la situación o aminorar sus efectos. El segundo, centrado en las emociones, tiene como función la regulación afectiva que involucra los esfuerzos para manejar el malestar y los estados emocionales resultantes del acontecimiento estresante. Estas últimas estrategias tienden a utilizarse en mayor medida cuando el sujeto ha realizado una evaluación del entorno y logra identificar que no puede hacer nada para modificar las condiciones desafiantes.

Entre investigaciones que evalúan las estrategias de afrontamiento usadas por víctimas del conflicto armado, se encuentra la de Hewitt Ramírez et al. (2016), quienes identificaron que las estrategias que más utilizaba una muestra de 677 sujetos eran la religión y la espera. Lo anterior coincide con los estudios realizados por Constanza Zuluaga (2016), Di-Colloredo, Aparicio Cruz y Moreno (2007) y Londoño Arredondo et al. (2008), quienes, al trabajar con población víctima del desplazamiento forzado, evidenciaron que en mayor medida los sujetos utilizaban la religión como estrategia de afrontamiento, además de la búsqueda de apoyo social y de apoyo profesional.

Otro determinante importante en el proceso de afrontamiento ante situaciones de alta demanda de estrés y frente a la recuperación tras eventos traumatizantes, como el conflicto armado, es la resiliencia, que es comprendida como aquella capacidad del ser humano para enfrentarse, sobreponerse y salir fortalecido y transformado ante situaciones adversas (Utria Utria, Amar Amar, Martínez González, Colmenares López \& Crespo Romero, 2015; Wilches, 2010). Para García Vesga y Domínguez-de la Ossa (2013), la resiliencia es vista como un proceso en el que median componentes familiares, comunitarios e individuales, los cuales determinan en gran medida la forma como se enfrentan y se superan las adversidades. Desde la psicología evolutiva, es vista como un proceso de desarrollo que les permite a las personas adaptarse positivamente, pese a las diferentes dificultades del entorno. No obstante, según Blanco Botero y Castro Molina (2013), hay personas que desarrollan estrategias resilientes, pese al daño, logrando resignificar sus vidas, mientras que en otros casos no se logra superar el dolor. Así, entonces, factores como el contexto, la edad, el género, la cultura y el nivel de escolaridad, al parecer, influirían sobre la resiliencia (Castillo Tenorio \& Palma Bernal, 2016), aunque para Cerquera Córdoba (2014) la resiliencia es una característica innata, es decir, propia de la personalidad, por ende, al ser esta una característica dinámica que depende de varios factores, se puede inferir que una persona puede ser resiliente en una situación particular y no serlo en otra. Por otro lado, para Becoña (2006) y Latorre Iglesias (2010), la resiliencia se aprende por medio del proceso de socialización primaria, es decir, existirían entornos que favorecen el cuidado y el buen trato en los primeros años de vida, potencializan el desarrollo de respuestas que mitigan el sufrimiento y fortalecen el desarrollo de capacidades para afrontar diversas crisis. Lo anterior es compartido por autores como Torres Gómez (2013), quien refiere 
que la capacidad para sobreponerse ante el dolor depende de variables personales y del contexto, así como, también, por el número, la intensidad y duración de las situaciones adversas por las que haya pasado el individuo.

Respecto de investigaciones que abordan la resiliencia en torno al conflicto armado en el contexto colombiano, Hewitt Ramírez et al. (2014, 2016) identificaron en su estudio, niveles de resiliencia en la muestra entre $32 \%$ y $61 \%$. Respecto del grupo de jóvenes, hallaron que altos niveles de resiliencia estaban asociados a la búsqueda de redes de apoyo. Por su parte, en la investigación de Domínguez de la Ossa y Godín Díaz (2007), a partir de la aplicación de una entrevista semiestructurada a víctimas en situación de desplazamiento, se encontró que la resiliencia era un factor característico en esta población, pues los sujetos referían tener proyectos por realizar, pese a las situaciones vividas. De igual manera, identificaron que los sujetos mantenían un interés por continuar en su proceso de conocimiento y reparación personal, identificando sus fortalezas y debilidades para salir adelante, así como, también reportaron que la fe en un ser supremo es un elemento de apoyo que permite la elaboración de sentido de vida. Lo anterior guarda relación con lo mencionado por Pérez Ruiz y Amorocho Cruz (2012), quienes señalan que pertenecer a una comunidad religiosa y llevar una vida espiritual son factores relevantes en el fortalecimiento de la resiliencia, pues promueve la búsqueda de apoyo y consuelo.

Cabe mencionar que en Colombia solo dos estudios abordan ambas variables (afrontamiento y resiliencia) en población víctima del conflicto armado; sin embargo, uno estudia las variables en una muestra de adultos con edad promedio de 43 años $(D E=33,43)$ (Hewitt Ramírez et al., 2016), mientras que el otro lo hace con una muestra de 289 niños con edades entre los 7 a 16 años de edad (edad promedio: 10 años) (Hewitt Ramírez et al., 2014). Por ello, explorar el comportamiento de estas variables en adultos jóvenes es una oportunidad para complementar y aportar a la comprensión del comportamiento y relación de estas variables en la población en cuestión.

Así, entonces, la presente investigación tuvo como objetivo analizar la relación entre las estrategias de afrontamiento y entre estas y el nivel de resiliencia presentado en una muestra de adultos jóvenes víctimas del conflicto armado colombiano.

\section{Método}

El presente estudio se realizó desde un enfoque cuantitativo de alcance descriptivo correlacional, no experimental y de corte transversal (Hernández Sampieri, Fernández Collado \& Baptista Lucio, 2014).

\section{Participantes}

En la investigación se trabajó con una muestra de 70 sujetos adultos jóvenes, víctimas del conflicto armado. Se implementó un muestreo no probabilístico por conveniencia en los municipios de Bucaramanga, Piedecuesta, Girón y Floridablanca de Colombia, siendo que las zonas lugares donde se llevó a cabo la aplicación de los instrumentos, son predominantemente habitados por personas víctimas y sobrevivientes desplazados por el conflicto armado quienes generalmente proceden de zonas rurales del país.

Vale mencionar que los criterios de inclusión fueron: (a) ser hombres y/o mujeres en rango de edad entre los 18 a 28 años, (b) tener capacidad y disponibilidad para responder a los cuestionarios, (c) aceptar la participación en la investigación a partir de la firma del consentimiento informado y (d) presentar documentación que garantiza la condición de víctima del conflicto armado por la unidad administrativa especial para la atención y reparación integral de las víctimas (Registro Único de Víctimas).

\section{Instrumentos}

Entrevista semiestructurada. Elaborada por los investigadores para indagar sobre variables sociodemográficas de la muestra seleccionada: género, edad, estado civil, nivel educativo y lugar de residencia.

Escala de Estrategias de Coping-Modificada (EEC-M) de Chorot y Sandín (1993) adaptada en Colombia por Londoño et al. (2006). El instrumento es autoadministrado y tiene como propósito identificar el uso de 12 estrategias de afrontamiento (ver Tabla 1). 
Tabla 1

Instrumento de estrategias de afrontamiento.

\begin{tabular}{|c|c|c|}
\hline Categoría de las estrategias & Descripción de la categoría & Ítems de la categoría \\
\hline Solución de problemas & $\begin{array}{l}\text { Consiste en el análisis de las causas del problema y la } \\
\text { búsqueda de soluciones alternativas }\end{array}$ & $10,17,19,26,28,37,39,51,68$ \\
\hline Búsqueda de apoyo social & $\begin{array}{l}\text { Se refiere a la búsqueda de apoyo por parte de amigos, } \\
\text { familiares y conocidos, siendo esta ayuda centrada en el } \\
\text { afecto, el apoyo emocional y suministro de información para } \\
\text { superar la situación problema }\end{array}$ & $6,14,23,24,34,47,57$ \\
\hline Espera & $\begin{array}{l}\text { Se basa en la consideración de que la situación problema se } \\
\text { resolverá por sí sola con el paso del tiempo, siendo una } \\
\text { estrategia cognitivo-conductual de tipo pasivo }\end{array}$ & $9,18,27,29,38,40,50,60,69$ \\
\hline Religión & $\begin{array}{l}\text { Relacionada con la creencia en un dios paternalista que } \\
\text { interviene por el individuo en las distintas situaciones } \\
\text { generadoras de estrés }\end{array}$ & $8,16,25,36,49,59,67$ \\
\hline Evitación emocional & $\begin{array}{l}\text { Consiste en la movilización de recursos para ocultar o inhibir } \\
\text { las propias reacciones emocionales consideradas como } \\
\text { negativas por el individuo }\end{array}$ & $11,20,21,30,41,43,53,62$ \\
\hline $\begin{array}{l}\text { Búsqueda de apoyo } \\
\text { profesional }\end{array}$ & $\begin{array}{l}\text { Considerada un tipo de apoyo social, se basa en la obtención } \\
\text { de información y alternativas de solución de cara a la } \\
\text { situación problema }\end{array}$ & $7,15,35,48,58$ \\
\hline Reacción agresiva & $\begin{array}{l}\text { Se refiere a la impulsiva expresión emocional dirigida a otros, } \\
\text { hacia sí mismo o incluso hacia los objetos, buscando con esto } \\
\text { restar carga emocional }\end{array}$ & $4,12,22,33,44$ \\
\hline Evitación cognitiva & $\begin{array}{l}\text { Basada en la pretensión de neutralizar pensamientos } \\
\text { negativos, para ello utiliza la desconexión mental, el } \\
\text { distanciamiento y la negación, caracterizándose entonces por } \\
\text { ser una estrategia de tipo pasivo, donde "la desconexión } \\
\text { mental se refiere al uso de pensamientos distractivos para } \\
\text { evitar pensar en la situación del problema. El } \\
\text { distanciamiento refiere la supresión cognitiva de los efectos } \\
\text { emocionales que el problema genera. La negación describe la } \\
\text { ausencia de aceptación del problema y su evitación por } \\
\text { distorsión o desfiguración del mismo en el momento de su } \\
\text { valoración" }\end{array}$ & $31,32,42,54,63$ \\
\hline $\begin{array}{l}\text { Expresión de la dificultad de } \\
\text { afrontamiento }\end{array}$ & $\begin{array}{l}\text { Se relaciona con la expresión de la dificultad a la hora de } \\
\text { expresar las emociones generadas por las situaciones } \\
\text { estresantes por lo que consiste en una muestra de } \\
\text { desesperanza frente al control de las emociones y el problema }\end{array}$ & $45,52,55,61$ \\
\hline Revaluación positiva & $\begin{array}{l}\text { Comprende la identificación y generación de aprendizaje a } \\
\text { partir de las situaciones enfrentadas, desde la óptica } \\
\text { optimista, consiste en la búsqueda y construcción de } \\
\text { interpretaciones sobre el valor que aporta al desarrollo } \\
\text { personal las situaciones estresantes, buscando que } \\
\text { percepción de amenaza sea atenuada a partir del cambio del } \\
\text { significado de la situación }\end{array}$ & $5,13,46,56,64$ \\
\hline Negación & $\begin{array}{l}\text { Consiste en la no aceptación del problema llevando a que el } \\
\text { comportamiento y el pensamiento eviten responder a la } \\
\text { situación problema }\end{array}$ & $1,2,3$ \\
\hline Autonomía & $\begin{array}{l}\text { Refiere de la tendencia a responder de manera independiente } \\
\text { a los problemas sin requerir el apoyo de otras personas }\end{array}$ & 65,66 \\
\hline
\end{tabular}

La escala cuenta con 69 ítems con respuesta en una escala tipo Likert: 1 (nunca), 2 (casi nunca), 3 (a veces), 4 (frecuentemente) 5 (casi siempre) y 6 (siempre). Para determinar si una estrategia es de mayor o menor uso, se tomó como referencia los valores de media, desviación estándar y percentiles de la EEC-M presentada por Londoño et al. (2006) en una muestra colombiana. En esta se establece que toda puntuación directa ubicada en el percentil 75 o por encima de él es considerada una estrategia con mayor uso. El instrumento presenta una consistencia interna, evaluada por alfa de Cronbach, de 0,847. En la muestra del presente estudio, el instrumento reporta un alfa de Cronbach de 0,748. Según los resultados de la medida KMO, la prueba de esfericidad de Bartlett y el análisis de comunalidad, el instrumento reporta validez para la presente muestra. 
Escala de Resiliencia (Resilience Scale, RS; Wagnild \& Young, 1993) adaptada en Perú por Novella (2002). La escala autoadministrada mide cinco dimensiones de la resiliencia (ver Tabla 2).

Tabla 2

Instrumento de resiliencia

\begin{tabular}{|c|c|c|}
\hline Categoría de las estrategias & Descripción de la categoría & Ítems de la categoría \\
\hline Ecuanimidad & $\begin{array}{l}\text { Denota una perspectiva balanceada } \\
\text { de la propia vida y experiencias, } \\
\text { tomar las cosas tranquilamente y } \\
\text { moderando las actitudes ante la } \\
\text { adversidad }\end{array}$ & $7,8,11,12$ \\
\hline Sentirse bien solo & $\begin{array}{l}\text { Nos da el significado de libertad y que } \\
\text { somos únicos y muy importantes }\end{array}$ & $5,3,19$ \\
\hline Confianza en sí mismo & $\begin{array}{l}\text { Habilidad para creer en sí mismo, en } \\
\text { sus capacidades }\end{array}$ & $6,9,10,13,17,18,24$ \\
\hline Perseverancia & $\begin{array}{l}\text { Persistencia ante la adversidad o el } \\
\text { desaliento, tener un fuerte deseo del } \\
\text { logro y autodisciplina }\end{array}$ & $1,2,4,14,15,20,23$ \\
\hline Satisfacción personal & $\begin{array}{l}\text { Comprender el significado personal } \\
\text { de la vida y como se contribuye a esta }\end{array}$ & $16,21,22,25$ \\
\hline
\end{tabular}

Tales dimensiones se distribuyen en 25 ítems escritos de forma positiva, con un sistema de respuesta tipo Likert de 7 puntos, desde 1 (en desacuerdo) a 7 (máximo acuerdo). La puntuación total varía de 25 a 175 puntos, siendo los más altos puntajes indicadores de mayor resiliencia. Presenta una consistencia interna, evaluada por alfa de Cronbach, de 0,89. En la muestra del presente estudio, el instrumento reporta un alfa de Cronbach de 0,956. Respecto de la validez, los resultados de la medida KMO, la prueba de esfericidad de Bartlett y el análisis de comunalidad reportan validez para la presente muestra.

Se consideraron altos niveles de resiliencia la media $+1 D E$ de la puntuación total reportada en la validación de Novella (2002), identificándose una media de 74,8 y una desviación de 20,1 en la muestra trabajada, para lo cual una puntuación alta se tomaría desde 94,9 puntos.

\section{Procedimiento}

Se realizó inicialmente un acercamiento a las unidades de atención y reparación a víctimas del conflicto armado en el departamento de Santander, Colombia, particularmente las ubicadas en los municipios de Bucaramanga, Floridablanca, Piedecuesta y Girón. A través de estos espacios para las víctimas, se contactó a los sujetos, quienes, de manera voluntaria y tras la explicación del objetivo de la investigación, manifestaban querer participar. Lo anterior permitió implementar el método de voz a voz entre diferentes líderes de grupos comunitarios y sujetos víctimas del conflicto armado.

Posteriormente al acercamiento a la población, se dio paso a la planeación para la aplicación de los instrumentos, los cuales se aplicaron entre mayo a julio de 2018, iniciando con la presentación y firma del consentimiento informado que se ampara bajo la Ley 1090 de 2006. El promedio de aplicación de las pruebas fue de 45 minutos a 1 hora.

\section{Análisis de Datos}

Se tabularon los datos y el análisis se realizó mediante el programa estadístico SPSS versión 22 . Se realizaron análisis descriptivos de las variables (frecuencias y porcentajes) y luego el análisis correlacional entre las propias estrategias de afrontamiento y entre estas y las categorías de resiliencia. Para ello se aplicó la prueba de normalidad de Kolmogorov-Smirnov, obteniendo una distribución normal de los datos, por tanto, se utilizó el coeficiente de correlación lineal de Pearson. Se utilizó también, la medida de KMO obteniendo en 
para la escala de Estrategias un puntaje de 0,669 y para la escala de resiliencia 0,882; aplicando además la prueba de esfericidad de Bartlett obteniendo para ambas pruebas un valor de significancia de $p<0,001$.

\section{Resultados}

En la Tabla 3 se puede apreciar que la mayoría de la muestra estuvo conformada por mujeres. El estrato o nivel socioeconómico (NSE) bajo predominó en la presente investigación, el rango de edad mayoritario fue de 22 a 26 años, los participantes eran mayoritariamente solteros y no habían finalizado el bachillerato.

Tabla 3

Datos Sociodemográficos de la Muestra de Adultos Jóvenes Victimas del Conflicto Armado

\begin{tabular}{lcr}
\hline \multicolumn{1}{c}{ Descriptor } & Frecuencia & $\%$ \\
\hline Sexo & & \\
$\quad$ Hombres & 23 & 32,9 \\
Mujeres & 47 & 67,1 \\
NSE & & \\
$\quad$ Estrato 1 & 24 & 34,3 \\
$\quad$ Estrato 2 & 46 & 65,7 \\
Rango de edad & & \\
16 años - 21 años & 14 & 20,0 \\
22 años- 26 años & 35 & 50,0 \\
27 años- 29 años & 21 & 30,0 \\
Estado civil & & \\
Casado & 9 & 12,9 \\
Unión libre & 6 & 8,5 \\
$\quad$ Soltero & 55 & 78,6 \\
Nivel de escolaridad & & \\
$\quad$ Primaria incompleta & 2 & 2,8 \\
Bachillerato sin terminar & 55 & 78,6 \\
$\quad$ Bachillerato completo & 13 & 18,6 \\
Lugar de residencia & & \\
$\quad$ Bucaramanga & 15 & 21,4 \\
$\quad$ Piedecuesta & 31 & 44,3 \\
$\quad$ Girón & 8 & 11,4 \\
$\quad$ Floridablanca & 16 & 22,9 \\
\hline
\end{tabular}

Nota. NSE: hace referencia a la estratificación de los usuarios según recursos económicos (estratos 1, 2 y 3 corresponden a estratos bajos). Respecto al descriptor lugar de residencia, se evidencian los cuatro municipios del departamento de Santander, Colombia.

$N=70$. Edad media $=24,9$ años. $D E=3,24$.

En la Tabla 4 se evidencia que las estrategias que con mayor frecuencia usa la muestra son la religión, la expresión de la dificultad de afrontamiento, la búsqueda de apoyo social y la reacción agresiva; mientras que las de menor uso fueron la búsqueda de apoyo profesional, la negación, la evitación cognitiva, la evitación emocional, la solución de problemas, la espera, la revaluación positiva y la autonomía. 
Tabla 4

Uso de Estrategias de Afrontamiento

\begin{tabular}{lcccc}
\hline \multirow{2}{*}{$\begin{array}{l}\text { Estrategia de afrontamiento } \\
\text { Solución de problemas }\end{array}$} & \multicolumn{2}{c}{ Mayor uso de la estrategia } & \multicolumn{2}{c}{ Menor uso de la estrategia } \\
\cline { 2 - 5 } & Frecuencia & Porcentaje & Frecuencia & Porcentaje \\
\hline Búsqueda de apoyo social & 15 & 21,4 & 55 & 78,6 \\
Espera & 54 & 77,1 & 16 & 22,9 \\
Religión & 20 & 28,6 & 50 & 71,4 \\
Evitación emocional & 62 & 88,6 & 8 & 11,4 \\
Búsqueda de apoyo profesional & 12 & 17,1 & 58 & 82,9 \\
Reacción agresiva & 3 & 4,3 & 67 & 95,7 \\
Evitación cognitiva & 48 & 68,6 & 22 & 31,4 \\
Revaluación positiva & 11 & 15,7 & 59 & 84,3 \\
Expresión de la dificultad de afrontamiento & 22 & 31,4 & 48 & 68,6 \\
Negación & 57 & 81,4 & 13 & 18,6 \\
Autonomía & 7 & 10,0 & 63 & 90,0 \\
\hline
\end{tabular}

En la Tabla 5 se puede apreciar que la mayoría de la muestra presentaba bajos niveles de resiliencia, mientras que solo una parte se encontraba en los rangos de normalidad y de altos niveles. Por otro lado, respecto a las categorías de resiliencia, se puede apreciar que la mayoría se encuentra en el nivel resiliencia bajo, como la satisfacción personal, la ecuanimidad y sentirse bien solos, mientras que la categoría de confianza en sí mismo y perseverancia se encuentran en los rangos de nivel de resiliencia normal.

Tabla 5

Nivel de Resiliencia: Total y Categorías

\begin{tabular}{llcc}
\hline \multicolumn{1}{c}{ Categoría } & \multicolumn{1}{c}{ Nivel } & Frecuencia & Porcentaje \\
\hline \multirow{3}{*}{ Total } & Resiliencia alta & 12 & 17,1 \\
& Resiliencia moderada & 18 & 25,7 \\
& Resiliencia baja & 40 & 57,1 \\
\hline \multirow{3}{*}{ Satisfacción personal } & Resiliencia alta & 13 & 18,6 \\
& Resiliencia moderada & 20 & 28,5 \\
& Resiliencia baja & 37 & 52,9 \\
\hline \multirow{3}{*}{ Ecuanimidad } & Resiliencia alta & 14 & 20,0 \\
& Resiliencia moderada & 17 & 24,3 \\
& Resiliencia baja & 39 & 55,7 \\
\hline \multirow{3}{*}{ Sentirse bien solo } & Resiliencia alta & 15 & 21,4 \\
& Resiliencia moderada & 17 & 24,3 \\
& Resiliencia baja & 38 & 54,3 \\
\hline \multirow{3}{*}{ Confianza en sí mismo } & Resiliencia alta & 15 & 21,4 \\
& Resiliencia moderada & 44 & 62,9 \\
& Resiliencia baja & 11 & 15,7 \\
\hline \multirow{2}{*}{ Perseverancia } & Resiliencia alta & 12 & 17,1 \\
& Resiliencia moderada & 35 & 50,0 \\
& Resiliencia baja & 23 & 32,9 \\
\hline
\end{tabular}

Se identificaron algunas correlaciones directas entre las estrategias de afrontamiento y las categorías de resiliencia (ver Tabla 6). Hubo correlaciones altas entre la estrategia de afrontamiento búsqueda de apoyo social y la categoría de resiliencia confianza en sí mismo; entre las estrategias evitación emocional y evitación cognitiva y las categorías de resiliencia ecuanimidad y sentirse bien solo; y, por último, la estrategia de afrontamiento revaluación positiva y las categorías de resiliencia satisfacción personal y ecuanimidad. 
Tabla 6

Correlacionales entre Estrategias de Afrontamiento y Categorias de Resiliencia

\begin{tabular}{|c|c|c|c|c|c|}
\hline \multirow[b]{2}{*}{ Estrategia de afrontamiento } & \multicolumn{5}{|c|}{ Categoría de resiliencia } \\
\hline & $\begin{array}{c}\text { Satisfacción } \\
\text { personal }\end{array}$ & Ecuanimidad & $\begin{array}{l}\text { Sentirse bien } \\
\text { solo }\end{array}$ & $\begin{array}{l}\text { Confianza en } \\
\text { sí mismo }\end{array}$ & Perseverancia \\
\hline Solución de problemas & $-0,059$ & $-0,111$ & $-0,353^{*}$ & $-0,454^{* *}$ & $-0,121$ \\
\hline Búsqueda apoyo social & 0,133 & $-0,061$ & 0,156 & $0,774^{* *}$ & $-0,018$ \\
\hline Espera & 0,175 & 0,020 & 0,127 & $0,376^{* *}$ & 0,104 \\
\hline Religión & $-0,030$ & $-0,064$ & 0,240 & 0,230 & $-0,012$ \\
\hline Evitación emocional & 0,029 & $0,773^{*}$ & $0,827^{*}$ & 0,276 & 0,182 \\
\hline Búsqueda de apoyo profesional & $-0,197$ & $-0,314^{*}$ & $-0,096$ & $-0,027$ & 0,028 \\
\hline Reacción agresiva & 0,005 & $-0,096$ & 0,217 & 0,315 & $-0,069$ \\
\hline Evitación cognitiva & $-0,158$ & $0,844^{*}$ & $0,845^{*}$ & $-0,342$ & $-0,056$ \\
\hline Revaluación positiva & $0,870^{*}$ & $0,766^{*}$ & $-0,147$ & $0,339^{*}$ & 0,048 \\
\hline $\begin{array}{l}\text { Expresión de la dificultad de } \\
\text { afrontamiento }\end{array}$ & 0,060 & 0,094 & 0,242 & $0,432^{* *}$ & 0,070 \\
\hline Negación & $-0,107$ & 0,022 & 0,005 & 0,012 & 0,127 \\
\hline Autonomía & 0,022 & $-0,099$ & $-0,211$ & $-0,300^{*}$ & $-0,002$ \\
\hline
\end{tabular}

${ }^{* *} p \leq 0,01 ; * p \leq 0,05$

Por último, en la Tabla 7 se pueden identificar correlaciones directas e inversas entre las propias categorías de estrategias de afrontamiento del instrumento. La estrategia solución de problemas correlacionó de manera inversa con búsqueda de apoyo social, reacción agresiva y expresión de la dificultad de afrontamiento; y de manera directa con la búsqueda de apoyo profesional, evitación cognitiva y revaluación positiva. En cuanto a la estrategia de búsqueda de apoyo social, se pudo identificar, además, una correlación inversa con evitación cognitiva, mientras que la religión correlaciona inversamente con evitación emocional. Respecto de la estrategia de evitación emocional, se pudo identificar que correlaciona inversamente, además, con la búsqueda de apoyo profesional, la reacción agresiva y la expresión de la dificultad de afrontamiento; y directamente con evitación cognitiva. En cuanto a la búsqueda de apoyo profesional, se identificaron, además, correlaciones inversas con reacción agresiva y con la expresión de la dificultad de afrontamiento; y una directa con la estrategia de revaluación positiva. Por otro lado, la estrategia de reacción agresiva correlaciona, además, directamente con expresión de la dificultad de afrontamiento e inversamente con evitación cognitiva y revaluación positiva. Con relación a la estrategia de evitación cognitiva, se pudo identificar, además, una correlación directa con revaluación positiva y una correlación inversa con expresión de la dificultad de afrontamiento. Por último, se encontró que la estrategia de revaluación positiva además correlacionó de manera inversa con expresión de la dificultad de afrontamiento.

\section{Discusión}

La muestra abordada para esta investigación fue conformada por hombres (33\%) y mujeres (67\%) reconocidos como víctimas del conflicto armado colombiano, en su mayoría solteros (78\%), que residen en estrato socioeconómico 1 y 2, con edades comprendidas entre los 16 a 28 años. Es necesario referir que el conflicto armado ha generado grandes afectaciones, especialmente a grupos poblacionales del sector rural (Andrade Salazar, Albarracín Ángel, Giraldo Giraldo \& Rico Ramos, 2012; Cerquera Córdoba, Peña Peña, García Vargas, Orejuela Castro \& García Nova, 2017; Comité Internacional de la Cruz Roja, 2010; Serpa Vega, 2013, Mayo 15), así como a mujeres, pues la alta prevalencia del género femenino en diversos estudios así lo demuestra (Alzate, Rico, Maza \& Sabucedo, 2018; Andrade Salazar et al., 2012; Andrade Salazar, Zuluaga Delgado, Ramírez Valladares \& Ramírez Hurtado, 2015; Campo-Arias et al., 2017; Hewitt Ramírez et al., 2016;). Sin embargo, existen investigaciones que reportan menor diferencia por género (CamargoVelásquez \& Palacio-Sañudo, 2017; Castaño et al., 2018; Lozano \& Gómez, 2004). 
Tabla 7

Correlacionales entre las Categorías de Estrategias de Afrontamiento

\begin{tabular}{|c|c|c|c|c|c|c|c|c|c|c|c|}
\hline & 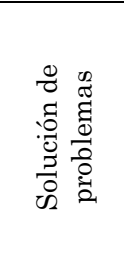 & 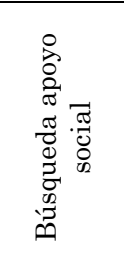 & 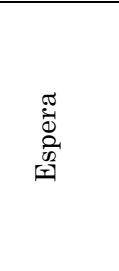 & 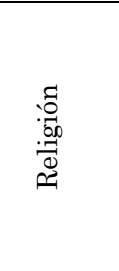 & 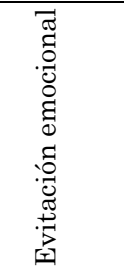 & 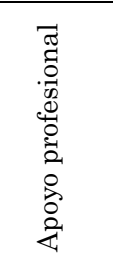 & 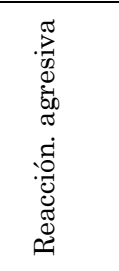 & 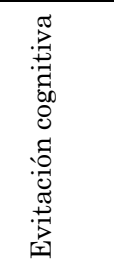 & 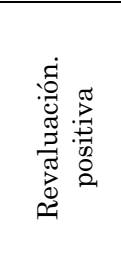 & 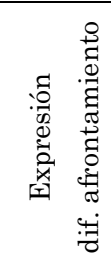 & 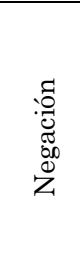 \\
\hline Búsqueda apoyo social & $-0,789^{* *}$ & & & & & & & & & & \\
\hline Espera & $-0,457$ & $0,425^{* *}$ & & & & & & & & & \\
\hline Religión & $-0,274$ & 0,176 & 0,130 & & & & & & & & \\
\hline Evitación emocional & $-0,405^{* *}$ & 0,106 & 0,258 & $0,655^{*}$ & & & & & & & \\
\hline Apoyo profesional & $0,588^{*}$ & $-0,044$ & $-0,278$ & 0,041 & $-0,684^{*}$ & & & & & & \\
\hline Reacción agresiva & $-0,629^{* *}$ & $0,346^{*}$ & $0,353^{*}$ & $0,421^{* *}$ & $-0,897^{* *}$ & $-0,527^{*}$ & & & & & \\
\hline Evitación cognitiva & $0,715^{*}$ & $-0,622 *$ & $-0,319$ & $-0,377^{* *}$ & $0,730 * *$ & $-0,061$ & $-0,839^{* *}$ & & & & \\
\hline Revaluación positiva & $0,596^{* *}$ & $-0,502$ & $-0,722^{* *}$ & $-0,324^{*}$ & $-0,483^{* *}$ & $0,745^{*}$ & $-0,619^{*}$ & $0,597^{*}$ & & & \\
\hline $\begin{array}{l}\text { Expresión dificultad } \\
\text { de afrontamiento }\end{array}$ & $-0,660^{* *}$ & $0,483^{* *}$ & 0,540 & 0,218 & $-0,850^{*}$ & $-0,697^{*}$ & $0,747^{* *}$ & $-0,622^{*}$ & $-0,733^{* *}$ & & \\
\hline Negación & 0,153 & $-0,267$ & 0,144 & $-0,306^{*}$ & 0,084 & $-0,249$ & $-0,235$ & $0,396^{* *}$ & $-0,249$ & 0,566 & \\
\hline Autonomía & $0,446^{* *}$ & $-0,439^{* *}$ & $-0,262$ & $-0,145$ & 0,014 & 0,073 & $-0,272$ & 0,443 & $-0,262$ & 0,344 & 0,153 \\
\hline
\end{tabular}

Lo anterior puede explicarse partiendo por reconocer que la ocurrencia de hechos que victimizan genera diversos daños y afectaciones a las personas y su núcleo familiar (Bello, 2013), siendo importante destacar que, entre estos, el desplazamiento forzado es el hecho más frecuente y que, a la par, en hechos como el homicidio, la desaparición forzada, el secuestro, la tortura, los actos terroristas y los accidentes por minas antipersonales, municiones sin explotar o artefactos explosivos improvisados, la población masculina es la principal víctima (RNI, 2019). Por ello, en investigaciones con víctimas del conflicto armado es habitual que las muestras sean conformadas mayoritariamente por mujeres, como en el presente estudio, puesto que con frecuencia, según Zorio (2015), son ellas las sobrevivientes de amenazas de las que fueron víctimas sus compañeros sentimentales: "si usted no me vende, me vende su viuda" (pp. 300-301). Esto ocasiona, en la gran mayoría de casos, que ellas asuman como jefas de hogar y se vean obligadas a cambiar su lugar de residencia (del rural al urbano).

En cuanto al hecho de que la totalidad de la muestra pertenezca al NSE 1 y 2, en similares estudios se expone que alrededor del $87,5 \%$ y el $91,1 \%$ de personas pertenecientes a las muestras pertenecen a estos NSE (Castaño et al., 2018; García Vargas \& Peña Peña, 2017). Tal situación se debe a que generalmente las víctimas del conflicto que residen en el sector urbano proceden del sector rural (Andrade Salazar et al., 2015; García Vargas \& Peña Peña, 2017; Tenjo, 2015), siendo habitual que los sobrevivientes no encuentren posibilidades laborales en el ámbito urbano, viéndose obligados a residir en sectores caracterizados por la baja cobertura de servicios públicos, con alta presencia de delincuencia y en condiciones de hacinamiento (Constanza Zuluaga, 2016; Guzmán Martínez, Ramírez Zambrano, Reyes Linares \& Varela Cardozo, 2013).

Por otra parte, la evaluación de estrategias de afrontamiento revela que la muestra usa con mayor frecuencia estrategias como la religión, la expresión de la dificultad de afrontamiento, la búsqueda de apoyo social y la reacción agresiva. Dichas estrategias corresponden, según Di-Colloredo et al. (2007), a estilos o actitudes de afrontamiento pasivo y enfocados en las emociones. Estos hallazgos resultan consecuentes con lo encontrado por Hewitt Ramírez et al. (2016), quienes señalan que en adultos $(N=677)$ expuestos al conflicto armado es frecuente el uso de las estrategias de afrontamiento como "esperar que las cosas se arreglen solas" y la religión. Incluso, Di-Colloredo et al. (2007) destacan que en población en situación de desplazamiento las mujeres usan la revaluación positiva de la situación, con tendencia al desarrollo personal, desconexión cognoscitiva del problema y distanciamiento de la situación, mientras que, en hombres, además del desarrollo y el control emocional, se develan marcadas las reacciones depresivas y de negación del problema (de la situación vivida y la actual). Una de las limitaciones de este estudio fue contar con una mayor 
muestra de mujeres, lo cual dificultó hacer un análisis comparativo a nivel de género. Es, por ende, posible que, al comparar las estrategias utilizadas por hombres y mujeres, se encuentren diferencias importantes.

Respecto del uso de diversas estrategias, es preciso señalar la existencia de resultados asociados en otras poblaciones. Por ejemplo, Chaves Castaño y Orozco Rosado (2015), al evaluar una muestra de 68 soldados, encuentran que la estrategia de afrontamiento más usada fue la espera. De igual manera, Amaya Guevara y Zacarías Mendoza (2017) examinan miembros de un comité de ex-presos políticos y algunos veteranos de guerra, encontrando que las estrategias de afrontamiento que más utilizan son el apoyo social y la religión. Incluso, respecto de la prevalencia de la reacción agresiva como una de las estrategias con mayor uso, Cobos Jiménez (2016) expone un hallazgo en una muestra de 151 mujeres víctimas de violencia de género, detectando que, a mayor gravedad de la violencia física mayor expresión emocional abierta.

En consecuencia, los estilos de afrontamiento señalados como predominantes en el presente estudio y que son semejantes a los identificados por otros estudios, dan cuenta de un estilo pasivo y/o centrado en las emociones, que implica cierto abandono o distanciamiento frente al problema, debido a una baja percepción de control sobre la situación, por lo cual resulta útil el ahorro de recursos conductuales, cognitivos y emocionales (Cobos Jiménez, 2016; Di-Colloredo et al., 2007).

Por otra parte, entre las estrategias de menor uso entre los participantes del presente estudio, se hallan el apoyo profesional, la negación, la evitación cognitiva, la evitación emocional, la revaluación positiva y otras centradas en el problema, como la solución de problemas, la espera y la autonomía. Lo anterior da cuenta de que los participantes no buscan apoyo profesional, a pesar de reconocer y aceptar la existencia del problema, no aprenden de las dificultades ni encuentran aspectos positivos en ellas, no ejecutan acciones para dar solución a los conflictos y, sin embargo, a la hora de responder a estos, buscan el apoyo de amigos y familiares, sin esperar que con el paso del tiempo la situación se resuelva de manera positiva (Gantiva Díaz, Luna Viveros, Dávila \& Salgado, 2010; Londoño Arredondo et al., 2008).

Lo señalado aquí da cuenta del bajo uso de estrategias racionales de afrontamiento, pues, tal como algunas investigaciones han identificado, estas se relacionan de forma inversa con la vivencia de violencia (Ceballos Ospino, Echeverri Arias \& Jiménez Villamizar, 2014; Cobos Jiménez, 2016). Estos precedentes podrían respaldar el hecho de que si en sobrevivientes del conflicto armado los hechos que los han victimizado han generado daños y afectaciones (físicas y mentales) que trascienden en el tiempo, dicha vivencia conexa a la experimentación de situaciones o condiciones de vulnerabilidad podría establecer o crear en la población modelos de estímulos neuronales de tipo nocivo (Traue, Jerg-Bretzke, Pfingsten \& Hrabal, 2010) que conllevarían a un estado de distrés general (García Vargas \& Peña Peña, 2017), inhabilitándoles para hacer una revaluación positiva de su situación.

De cara a lo anterior, estos hallazgos permiten señalar que las intervenciones (grupales o individuales) con esta población deben incluir dentro de sus objetivos la diversificación de las estrategias de afrontamiento usadas, promover estilos más activos e incentivar la resignificación de las situaciones (pasadas y actuales), buscando aumentar la percepción de control, reactivar y empoderar a las víctimas frente a su situación actual, para lo cual se considera altamente efectiva la vinculación o el trabajo con grupos de apoyo (familiar y social) y de orden multidisciplinario.

En cuanto a la resiliencia, el presente estudio indica que el $57 \%$ de la muestra presenta un nivel bajo de resiliencia, mientras que el $26 \%$ refleja un rango moderado y el $17 \%$ presenta un alto nivel de resiliencia. En contraste a estos resultados, Hewitt Ramírez et al. (2016) manifiestan que el $61 \%$ de la población expuesta al conflicto armado $(N=677)$ tiene un nivel moderado de resiliencia, mientras que un $32 \%$ presenta un nivel alto. De igual manera lo reportan Aguilar Monsalve, Henao Ortiz, Tezón y Ladrón de Guevara Vásquez (2017), quienes afirman que, en una muestra de 31 víctimas del conflicto armado, el $97 \%$ es resiliente y el $3 \%$ tiene una resiliencia moderada; sin embargo y en contraste, Cobos Jiménez (2016) encuentra que en una muestra de mujeres víctimas de violencia de género, la mayoría no obtiene puntuaciones altas en resiliencia $(69 \%, N=134)$.

Reconociendo, por tanto, que las víctimas han vivido una historia de desestabilización familiar, que genera desarraigos, traumas, deterioros de identidad y bajo sentido de pertenencia (González Ocampo \& Bedmar Moreno, 2012), lo cual se relaciona con la constante experimentación de miedo, nostalgia, odio, rabia y tristeza, y en donde aparece el silencio y el aislamiento (López López, Pérez Durán \& Pineda-Marin, 2016); es viable considerar que estas condiciones ligadas a la vulnerabilidad social (Andrade Salazar et al., 2012; Campo-Arias et al., 2017) confluyan y generen un ambiente o contexto que limita la capacidad de los 
individuos para proyectarse en el futuro, para enfrentar más adversidades, para verse fortalecidos y transformados positivamente tras situaciones problemáticas, en pocas palabras, para ser resilientes.

Ahora bien, con relación a los resultados de las correlaciones entre las estrategias de afrontamiento y la resiliencia, estos reflejan que existe relación especialmente cuando las estrategias usadas por los sujetos están centradas o enfocadas en las emociones, siendo la ecuanimidad la más implicada en la relación con el afrontamiento, por ende, es factible considerar que tal tendencia a mantener una perspectiva balanceada de la propia vida y experiencias es el reflejo de una actitud pasiva de adaptación a las situaciones, pues se asocia a estrategias no centradas en el problema, lo cual es característico de las personas menos resilientes (Connor, 2006; González Torres \& Artuch Garde, 2014), puesto que se ha señalado que el afrontamiento activo promueve la resiliencia, mientras que el pasivo la inhibe (Yoon, Lee, Lee, Cho \& Lee, 2014).

Respecto de la estrategia de solución de problemas, se encontraron correlaciones con la reacción agresiva (inversa) y la revaluación positiva (directa). Para autores como Rojas de Escalona (2010), la eficacia y eficiencia para resolver los problemas están mediadas por la capacidad para procesar, planear y comprender la problemática. Estos factores influyen significativamente, ya que la representación que tenga la persona sobre el problema determina la manera en cómo planeará resolverlo. Asimismo, refieren que dificultades en la capacidad para resolver problemas trae consigo mayores niveles de frustración, lo cual puede influir en la implementación de estrategias de tipo agresivo, como se puede apreciar en los resultados encontrados en la investigación.

Lo anterior guarda relación con los postulados de Arán-Filippetti y Richaud de Minzi (2011), quienes refieren que sujetos que tienden a la impulsividad como mecanismo de afrontamiento presentan dificultades para resolver tareas, por la misma precipitación de sus respuestas, por el poco control atencional y emocional que presenta y por el inadecuado uso de estrategias de tipo analítico. Esta condición, a su vez, influye notoriamente en la capacidad para revaluar positivamente las adversidades, ya que, como lo mencionan Watzlawick (1995) y Weisberg (1989), ambos citados en Rojas de Escalona (2010), la capacidad de resolución de problemas supone una restructuración que implica cambiar el marco conceptual en su interpretación del problema, la manera como se piensa sobre este y sobre sí mismo y el aspecto emocional relacionado a la manera en cómo se siente el individuo en función del problema. Este cambio de sentido permite la apertura de nuevas alternativas de respuestas de afrontamiento.

Con relación a la estrategia de afrontamiento de búsqueda de apoyo social, se pudo identificar que el bajo uso de la evitación cognitiva tiene que ver con el exceso de rumiación de pensamientos, que en este caso resultan negativos, dado que los contenidos de los mismos se vuelven reiterativos respecto a las experiencias vividas en el conflicto armado. Pareciera, entonces, que las redes de apoyo para los sujetos de la presente investigación contribuyen en el desarrollo de estos estilos sostenidos de pensamiento, ya que, movidos por la necesidad de supervivencia y de aceptación social, tejen redes con sus pares cercanos que presentan las mismas situaciones de vulnerabilidad que ellos. Quizás, esto se deba a la condición de desplazamiento con relación a la zona geográfica en la que se encuentra actualmente la población, ya que gran parte de la muestra vive en asentamientos humanos que se caracterizan por presentar altos niveles de delincuencia y violencia (Constanza Zuluaga, 2016; Guzmán Martínez et al., 2013), aspectos que podrían fomentar la implementación de estrategias de tipo agresivo y reactivo.

También se logró identificar una correlación inversa entre evitación emocional y reacción agresiva, frente a esto, cabe destacar que, según Londoño et al. (2006), la evitación emocional es una estrategia cognitivo comportamental en la cual se evita expresar emociones, dada la carga emocional que representan. Evidenciar el poco uso de esta estrategia se considera como un factor de riesgo en la muestra, ya que, como lo refieren Serrano y García Alva (2010), la falta de control afectivo o poco desarrollo de inteligencia emocional puede traer consigo el desarrollo de trastornos psicológicos, como la depresión o la ansiedad o en el manejo de la ira. Lo anterior guarda relación con algunos criterios diagnósticos para los trastornos depresivos y otros trastornos relacionados (American Psychiatric Asociation, 2013).

Asimismo, otro factor de riesgo al presentar bajos niveles en la estrategia de afrontamiento de evitación emocional es el poco uso de la estrategia de evitación cognitiva, pues, según Londoño et al. (2006), altos niveles en esta estrategia están relacionados con la capacidad del sujeto para neutralizar sus pensamientos valorados como negativos o perturbadores, por medio de la distracción y la actividad, mientras que bajos niveles están asociados al exceso de pensamiento rumiativo negativo. Según Nolen-Hoeksema y Aldao (2011), entre los factores relacionados a la aparición, mantenimiento y recurrencia de la depresión, la rumiación (Watkins, 2008) ha cobrado importancia a la hora de entender las experiencias depresivas. Nolen-Hoeksema 
(1991, citado en García Cruz, Valencia Ortiz, Hernández-Martínez y Rocha Sánchez, 2017) refiere que la rumiación aparece como una forma de afrontar la tristeza, por lo que se puede constituir como una forma de afrontamiento que, por sus características, tiende a ser desadaptativa, dado que el sujeto tiende a pensar de manera reiterativa sobre las situaciones negativas, sin dar solución. Lo anterior cobra mayor relevancia al identificarse que en diferentes investigaciones se ha corroborado que este tipo de estrategia es un predictor y un factor que exacerba las psicopatologías de trastornos del estado de ánimo y depresión severa (Abela \& Hankin, 2009; Nolen-Hoeksema, Wisco \& Lyubomirsky, 2008).

Para culminar, en cuanto a limitaciones del presente estudio, aunque este se enfocó en una muestra de víctimas, se considera como limitación el no haber incluido una muestra de no víctimas, pues es viable y pertinente comparar las dos muestras con el ánimo de explorar si existen diferencias susceptibles de análisis. Ya se señaló la limitación respecto de una mayor submuestra de mujeres.

\section{Referencias}

Abela, J. R. Z. \& Hankin, B. L. (2009). Cognitive vulnerability to depression in adolescents: A developmental psychopathology perspective. En S. Nolen-Hoeksema y L. Hilt (Ed.), Handbook of depression in adolescents (pp. 336-363). New York, NY: Routledge.

Aguilar Monsalve, J., Henao Ortiz, M., Tezón, M. \& Ladrón de Guevara Vásquez, C. I. (2017). Relación entre la resiliencia y la capacidad de perdón como componentes psicosociales en adultos víctimas del conflicto armado de la comunidad de Zipacoa. Hexágono Pedagógico, 8(1), 133-154. https://doi.org/10.22519/2145888X.1072

Alejo, E. G., Rueda, G., Ortega, M. \& Orozco, L. C. (2007). Estudio epidemiológico del trastorno por estrés postraumático en población desplazada por la violencia política en Colombia. Universitas Psychologica, 6, 623-635. Extraído de http://www.scielo.org.co/pdf/rups/v6n3/v6n3a14.pdf

Alzate, M., Rico, D., Maza, M. \& Sabucedo, J. M. (2018). Dimensiones socioemocional e instrumental de la reconciliación social en el conflicto armado colombiano. Revista de Estudios Sociales, 66, 81-91. https://doi.org/10.7440/res66.2018.08

Amaya Guevara, D. C. \& Zacarías Mendoza, C. B. (2017). La funcionalidad familiar y su relación con las estrategias de afrontamiento al estrés utilizadas por sobrevivientes del pasado conflicto armado (Tesis de Grado, Universidad de El Salvador, San Salvador, El Salvador). Extraído de http://ri.ues.edu.sv/15674/1/14103278.pdf

American Psychiatric Association (2013). Diagnostic and statistical manual of mental disorders: DSM-5 (5a ed.). Arlington, VA: Autor. https://doi.org/10.1176/appi.books.9780890425596

Andrade Salazar, J. A., Albarracín Ángel, P. A., Giraldo Giraldo, J. E. \& Rico Ramos, A. (2012). Razones para vivir en personas que se encuentran en situación de desplazamiento forzado. Revista Colombiana de Ciencias Sociales, 3, 198-210. Extraído de https://dialnet.unirioja.es/descarga/articulo/5123803.pdf

Andrade Salazar, J. A., Zuluaga Delgado, C. V., Ramírez Valladares, J. J. \& Ramírez Hurtado, M. A. (2015). Caracterización y motivos para desplazarse en familias víctimas de conflicto armado asentadas en el departamento del Quindío. Pensamiento Americano, 8(15), 13-26. Extraído de https://publicaciones.americana.edu.co/index.php/pensamientoamericano/article/view/76/94

Arán-Filippetti, V. \& Richaud de Minzi, M. C. (2011). Efectos de un programa de intervención para aumentar la reflexividad y la planificación en un ámbito escolar de alto riesgo por pobreza. Universitas Psychologica, 10, 341-354. https://doi.org/10.11144/Javeriana.upsy10-2.epia

Becoña, E. (2006). Resiliencia: definición, características y utilidad del concepto. Revista de Psicopatología y Psicología Clínica, 11, 125146. https://doi.org/10.5944/rppc.vol.11.num.3.2006.4024

Bell, V., Méndez, F., Martínez, C., Palma, P. P. \& Bosch, M. (2012). Characteristics of the Colombian armed conflict and the mental health of civilians living in active conflict zones. Conflict and Health, 6, artículo 10. https://doi.org/10.1186/1752-1505-6-10

Sánchez, G. \& Bello, M. N. (Coord.) (2013). ;Basta ya! Colombia: memorias de guerra y dignidad. Bogotá, Colombia: Centro Nacional de Memoria Histórica. Extraído de http://www.centrodememoriahistorica.gov.co/descargas/informes2013/bastaYa/basta-ya-colombiamemorias-de-guerra-y-dignidad-2016.pdf

Blanco Botero, C. A. \& Castro Molina, K. P. (2013). Memoria, didáctica y resiliencia: un estudio cualitativo en la población de Nueva Venecia, departamento del Magdalena (2009-2011) (Tesis de Magíster, Universidad Sergio Arboleda, Bogotá, Colombia). Extraído de http://repositorio.ub.edu.ar/handle/123456789/2058

Camargo-Velásquez, A. \& Palacio-Sañudo, J. (2017). Apoyo social y sentido de comunidad en desplazados y damnificados en el departamento del Magdalena. Duazary: Revista Internacional de Ciencias de la Salud, 14, 35-44. https://doi.org/10.21676/2389783X.1735

Campo-Arias, A., Ospino, A. C., Sanabria, A. R., Guerra, V. M., Caamaño, B. H. \& Herazo, E. (2017). Frecuencia y variables asociadas a estigma-discriminación percibido en víctimas del conflicto armado colombiano. Cadernos de Saúde Pública, 33(11), 1-6. https://doi.org/10.1590/0102-311x00001117

Castaño, G., Sierra, G., Sánchez, D., Torres, Y., Salas, C. \& Buitrago, C. (2018). Trastornos mentales y consumo de drogas en población víctima del conflicto armado en tres ciudades de Colombia. Biomédica, 38(Suplemento 1: Enfermedades Crónicas), 77-92. https://doi.org/10.7705/biomedica.v38i0.3890

Castillo Tenorio, A. \& Palma Bernal, E. (2016). Resiliencia en mujeres viudas por el conflicto armado vinculadas a FUNVIDA en Tumaco (Tesis de Magíster, Pontificia Universidad Javeriana, Santiago de Cali, Colombia). Extraído de http://vitela.javerianacali.edu.co/handle/11522/7313

Ceballos Ospino, G. A., Echeverri Arias, A. \& Jiménez Villamizar, M. P. (2014). Uso de estrategias de afrontamiento de los pacientes con el VIH y el cáncer en una clínica privada en Santa Marta - Colombia. Revista Facultad de Salud, 6(1), 27-33. https://doi.org/10.25054/rfs.v6i1.150

Cerquera Córdoba, A. M. (2014). Programa de intervención psicológica en resiliencia para cuidadores informales de pacientes con demencia tipo Alzheimer en Bucaramanga, Colombia (PIRCA) (Tesis de Doctorado no publicada), Universidad de La Habana, Cuba.

Cerquera Córdoba, A. M., Peña Peña, A. J., García Vargas, C. J., Orejuela Castro, D. A. \& García Nova, M. A. (2017). Psicología de la salud en el contexto del conflicto armado en Colombia: un marco de referencia. Psicogente, 20, 181-191. https://doi.org/10.17081/psico.20.37.2427 
Chaves Castaño, L. \& Orozco Rosado, A. M. (2015). Estilos de personalidad y estrategias de afrontamiento al estrés en soldados: un estudio correlacional. Revista de Psicología Universidad de Antioquia, 7(1), 35-56. Extraído de http://aprendeenlinea.udea.edu.co/revistas/index.php/psicologia/article/view/25262/20872

Chorot, P. \& Sandín, B. (1993). Escala de Estrategias de Coping Revisado (EEC-R). Madrid: UNED

Cobos Jiménez, E. (2016). Apego, resiliencia y afrontamiento: un estudio con víctimas de violencia de género (Tesis de Doctorado, Universidad Complutense de Madrid, España). Extraído de https://eprints.ucm.es/38848/1/T37657.pdf

Comité Internacional de la Cruz Roja (2010). Colombia: consecuencias humanitarias del conflicto armado en Colombia. Informe. Genève, Suiza: Autor. Extraído de https://www.icrc.org/es/resources/documents/report/colombia-report-intro-220410.htm

Connor, K. M. (2006). Assesment of resilience in the aftermath of trauma. Journal of Clinical Psychiatry, 67(Supplement 2), 46-49. Extraído de http://www.repar.veille.qc.ca/info-tcc/IMG/pdf/Connor_2006.pdf

Constanza Zuluaga, L. (2016). Estrategias de afrontamiento en un grupo de desplazados internos en la ciudad de Bogotá. Revista de Psicología Universidad de Antioquia, 8(2), 71-86. https://doi.org/10.17533/udea.rpsua.v8n2a05

Corporación Acompañamiento Psicosocial y Atención en Salud Mental a Víctimas de Violencia Política (2008). Acompañamiento psicosocial con víctimas del desplazamiento forzado fundamentado en los derechos humanos. Bogotá, Colombia. Extraído de http://www.corporacionavre.org/wp-content/uploads/2015/03/ACOMPAN\%CC\%83AMIENTO-PSICOSOCIAL-CONVI\%CC\%81CTIMAS-DEL-DESPLAZAMIENTO-FORZADO-FUNDAMENTANDO-EN-LOS-DERECHOS-HUMANOS.pdf

Di-Colloredo, C., Aparicio Cruz, D. P. \& Moreno, J. (2007). Descripción de los estilos de afrontamiento en hombres y mujeres ante la situación de desplazamiento. Psychologia: Avances de la Disciplina, 2(1), 125-156. Extraído de https://www.redalyc.org//pdf/2972/297224996002.pdf

Domínguez de la Ossa, E. \& Godín Díaz, R. (2007). La resiliencia en familias desplazadas por la violencia sociopolítica ubicadas en Sincelejo. Psicología desde el Caribe, 19, 154-159. Extraído de http://www.redalyc.org/articulo.oa?id=21301907

Gantiva Díaz, C. A., Luna Viveros, A., Dávila, A. M. \& Salgado, M. J. (2010). Estrategias de afrontamiento en personas con ansiedad. Psychologia: Avances de la Disciplina, 4(1), 63-70. Extraído de http://www.redalyc.org/pdf/2972/297224086006.pdf

García Cruz, R., Valencia Ortiz, A. I., Hernández-Martínez, A. \& Rocha Sánchez, T. E. (2017). Pensamiento rumiativo y depresión entre estudiantes universitarios: repensando el impacto del género. Interamerican Journal of Psychology, 51, 406-416. Extraído de http://www.redalyc.org/pdf/284/28455448011.pdf

García Vargas, C. J. \& Peña Peña, A. J. (2017). Dolor, funcionalidad familiar y apoyo social en víctimas del conflicto armado colombiano con deficiencias en estructuras corporales (Tesis de Grado, Universidad Pontificia Bolivariana, Bucamaranga, Colombia). Extraído de https://biblioteca.bucaramanga.upb.edu.co/docs/digital_35527.pdf

García-Vesga, M. C. \& Domínguez-de la Ossa, E. (2013). Desarrollo teórico de la resiliencia y su aplicación en situaciones adversas: una revisión analítica. Revista Latinoamericana de Ciencias Sociales, Niñez y Juventud, 11, 63-77. Extraído de http://www.redalyc.org/articulo.oa?id=77325885001

González Ocampo, L. H. \& Bedmar Moreno, M. (2012). Población infantil en situación de desplazamiento forzado en Colombia y sus manifestaciones de ciudadanía. Revista de Paz y Conflictos, 5, 120-137. Extraído de https://www.ugr.es/ revpaz/articulos/rpc_n5_2012_art6.pdf

González Torres, M. C. \& Artuch Garde, R. (2014). Perfiles de resiliencia y estrategias de afrontamiento en la universidad: variables contextuales y demográficas. Electronic Journal of Research in Educational Psychology, 12, 621-648. https://doi.org/10.14204/ejrep.34.14032

Guzmán Martínez, L. F., Ramírez Zambrano, J. P., Reyes Linares, L. F. \& Varela Cardozo, L. (2013). Dinámicas de las familias vinculadas a la mesa de participación de víctimas del conflicto armado adscrita a la subdirección local para la integración social de Usme-Sumapaz (Tesis de Grado, Universidad de La Salle, Bogotá, Colombia). Extraído de https://ciencia.lasalle.edu.co/trabajo_social/179

Hernández Sampieri, R., Fernández Collado, C. \& Baptista Lucio, P. (2014). Metodología de investigación (6ª ed.). México DF, México: McGraw Hill.

Hewitt Ramírez, N., Gantiva Díaz, C. A., Vera Maldonado, A., Cuervo Rodríguez, M. P., Hernández Olaya, N. L., Juárez, F. \& Parada Baños, A. J. (2014). Afectaciones psicológicas de niños y adolescentes expuestos al conflicto armado en una zona rural de Colombia. Acta Colombiana de Psicología, 17(1), 79-89. https:// doi.org/10.14718/ACP.2014.17.1.9

Hewitt Ramírez, N., Juárez, F., Parada Baños, A. J., Guerrero Luzardo, J., Romero Chávez, Y. M., Salgado Castilla, A. M. \& Vargas Amaya, M. V. (2016). Afectaciones psicológicas, estrategias de afrontamiento y niveles de resiliencia de adultos expuestos al conflicto armado en Colombia. Revista Colombiana de Psicología, 25, 125-140. https://doi.org/10.15446/rcp.v25n1.49966

Huijts, I., Kleijn, W. C., van Emmerik, A. A. P., Noordhof, A. \& Smith, A. J. M. (2012). Dealing with man-made trauma: The relationship between coping style, posttraumatic stress, and quality of life in resettled, traumatized refugees in the Netherlands. Journal of Traumatic Stress, 25, 71-78. https://doi.org/10.1002/jts.21665

Husain, F., Mark, A., Lopes Cardozo, B., Becknell, K., Blanton, C., Araki, D. \& Vithana, E. K. (2011). Prevalence of war-related mental health conditions and association with displacement status in postwar Jaffna district, Sri Lanka. The Journal of the American Medical Association, 306, 522-531. https://doi.org/10.1001/jama.2011.1052

Latorre Iglesias, E. L. (2010). Memoria y resiliencia. Estudio de la memoria de las víctimas del conflicto armado en el departamento del Magdalena: presentificación, visibilización, catarsis y resiliencia. Prolegómenos: Derechos y Valores, 13(25), 95-109.

Ministerio de Salud y Protección Social. Ley 1090 de 2006. Recuperado de: https://www.unisabana.edu.co/fileadmin/Archivos_de_usuario/Documentos/Documentos_Investigacion/Docs_Comite_Etica/Ley_1090_ 2006_-_Psicologia_unisabana.pdf

Lazarus, R. S. \& Folkman, S. (1986). Estrés y procesos cognitivos. Barcelona, España: Martínez Roca.

Lira, E. (2010). Trauma, duelo, reparación y memoria. Revista de Estudios Sociales, 36, 14-28. Extraído de http://www.scielo.org.co/pdf/res/n36/n36a02.pdf

Londoño, A., Romero, P. \& Casas, G. (2012). The association between armed conflict, violence, and mental health: A cross sectional study comparing two populations in Cundinamarca department, Colombia. Conflict and Health, 6, artículo 12. https://doi.org/10.1186/1752-1505-6-12

Londoño Arredondo, N. H., Patiño Gaviria, C. D., Restrepo Ochoa, D. A., Correa Uribe, J. E., Raigoza Cardona, J., Toro Mondragón, L. ... Rojas, C. (2008). Perfil cognitivo asociado al trastorno por estrés postraumático en víctimas de violencia armada. Informes Psicológicos, 10, 11-27. Extraído de https://revistas.upb.edu.co/index.php/informespsicologicos/article/view/1694/1638 
Londoño, N. H., Henao López, G. C., Puerta, I. C., Posada, S., Arango, D. \& Aguirre-Acevedo, D. C. (2006). Propiedades psicométricas y validación de la Escala de Estrategias de Coping Modificada (EEC-M) en una muestra colombiana. Universitas Psychologica, 5, 327-349. Extraído de http://www.scielo.org.co/pdf/rups/v5n2/v5n2a10.pdf

López López, W., Pérez Durán, C. \& Pineda-Marin, C. (2016). Relación entre el perdón, la reconciliación y la salud mental de las víctimas de la violencia socio-política. Journal of Victimology, 3, 141-159. https://doi.org/10.12827/RVJV.3.06

Lozano, M. C. \& Gómez, M. L. (2004). Aspectos psicológicos, sociales y jurídicos del desplazamiento forzoso en Colombia. Acta Colombiana de Psicología, 12, 103-119. Extraído de https://editorial.ucatolica.edu.co/index.php/acta-colombianapsicologia/article/view/473/470

McDonald, L. (2010). Psychosocial rehabilitation of civilians in conflict-affected settings. En E. Martz (Ed.), Trauma rehabilitation after war and conflict: Community and individual perspectives (pp. 215-245). New York, NY: Springer. https://doi.org/10.1007/978-14419-5722-1_10

Moreno Camacho, M. A. \& Díaz Rico, M. E. (2016). Posturas en la atención psicosocial a víctimas del conflicto armado en Colombia. El Ágora USB, 16, 193-213. https://doi.org/10.21500/16578031.2172

Nolen-Hoeksema, S. \& Aldao, A. (2011). Gender and age differences in emotion regulation strategies and their relationship to depressive symptoms. Personality and Individual Differences, 51, 704-708. https://doi.org/10.1016/j.paid.2011.06.012

Nolen-Hoeksema, S., Wisco, B. E. \& Lyubomirsky, S. (2008). Rethinking rumination. Perspectives on Psychological Science, 3, $400-424$. https://doi.org/10.1111/j.1745-6924.2008.00088.x

Novella, A. (2002). Incremento de la resiliencia luego de la aplicación de un programa de psicoterapia breve en madres adolescentes (Tesis de Magíster no publicada, Universidad Nacional Mayor de San Marcos, Lima, Perú).

Pérez Ruiz, L. P. \& Amorocho Cruz, M. P. (2012). Resiliencia y desplazamiento y en niñas y niños a través del arte (Tesis de Grado, Pontificia Universidad Javeriana, Bogotá, Colombia). Extraído de https://repository.javeriana.edu.co/handle/10554/12291

Red Nacional de Información (2019). Registro único de víctimas. Bogotá, Colombia. Extraído de https://www.unidadvictimas.gov.co/es/registro-unico-de-victimas-ruv/37394

Rodríguez Díaz, M. C. (2006). Eficacia de un programa de grupo estructurado en estrategias de afrontamiento para DSTP en adultos y adolescentes en situación de desplazamiento. Universitas Psychologica, 5, 259-274. Extraído de http://www.scielo.org.co/pdf/rups/v5n2/v5n2a05.pdf

Rojas de Escalona, B. (2010). Solución de problemas: una estrategia para la evaluación del pensamiento creativo. Sapiens: Revista Universitaria de Investigación, 11(1), 117-125. Extraído de https://www.redalyc.org/pdf/410/41021794008.pdf

Schweitzer, R., Greenslade, J. \& Kagee, A. (2007). Coping and resilience in refugees from Sudan: A narrative account. Australian and New Zealand Journal of Psychiatry, 41, 282-288. https://doi.org/10.1080/00048670601172780

Serpa Vega, C. J. (2013, Mayo 15). Conflicto armado y post-conflicto. El Heraldo, Blogs, Política. Extraído de https://www.elheraldo.coconflicto-armado-y-post-conflicto-110408

Serrano, M. E. \& García Alva, D. (2010). Inteligencia emocional: autocontrol en adolescentes estudiantes de último año de secundaria. Multiciencias, 10, 273-280. Extraído de http://www.redalyc.org/articulo.oa?id=90416328008

Steel, Z., Chey, T., Silove, D., Marnane, C., Bryant, R. A. \& van Ommeren, M. (2009). Association of torture and other potentially traumatic events with mental health outcomes among populations exposed to mass conflict and displacement: A systematic review and meta-analysis. The Journal of the American Medical Association, 302, 537-549. https://doi.org/10.1001/jama.2009.1132

Tenjo, J. (2015). El mercado laboral rural en 2013. En J. Tenjo, Mercado laboral en el sector rural colombiano (pp. 8-17). Bogotá, Colombia: Departamento Nacional de Planeación, Misión para la Transformación del Campo. Extraído de https://colaboracion.dnp.gov.co/CDT/Agriculturapecuarioforestal\%20y\%20pesca/Mercado\%20Laboral\%20Rural.pdf

Torres Gómez, F. (2013). Intervención profesional desde la consultoría con enfoque resiliente en familias víctimas del conflicto armado. Tendencias \& Retos, 18(1), 33-48. Extraído de https://dialnet.unirioja.es/descarga/articulo/4929402.pdf

Traue, H. C., Jerg-Bretzke, L., Pfingsten, M. \& Hrabal, V. (2010). Factores psicológicos del dolor crónico. En A. Kopf \& N. B. Patel (Eds.), Guía para el manejo del dolor en condiciones de bajos recursos (pp. 19-26). Washington, DC: Asociación Internacional para el Estudio del Dolor. Extraído de https://www.iasp-pain.org/files/Content/ContentFolders/Publications2/FreeBooks/GuidetoPainManagement_Spanish.pdf

Utria Utria, L., Amar Amar, J. J., Martínez González, M., Colmenares López, G. \& Crespo Romero, F. (2015). Resiliencia en mujeres víctimas del desplazamiento forzado. Barranquilla, Colombia: Editorial Universidad del Norte.

Wagnild, G. M., \& Young, H. (1993). Development and psychometric. Journal of nursing measurement, 1(2), 165-178. Extraído de https://cyberleninka.org/article/n/255719.pdf

Watkins, E. R. (2008). Constructive and unconstructive repetitive thought. Psychological Bulletin, 134, 163-206. https://doi.org/10.1037/0033-2909.134.2.163

Wilches, I. (2010). Lo que hemos aprendido sobre la atención a mujeres víctimas de violencia sexual en el conflicto armado colombiano. Revista de Estudios Sociales, 36, 86-94. Extraído de www.redalyc.org/articulo.oa?id=81514696008

Yoon, J. H., Lee, J. H., Lee, C. Y., Cho, M. \& Lee, S. M. (2014). Suppressor effects of coping strategies on resilience. Asia Pacific Education Review, 15, 537-545. https://doi.org/10.1007/s12564-014-9343-8

Zorio, S. (2015). Tierras, mujeres y niñez. Familia y conflicto armado. Revista Derecho del Estado, 35, $295-315$. https://doi.org/10.18601/01229893.n35.11.

Fecha de recepción: Noviembre de 2018.

Fecha de aceptación: Octubre de 2019. 